\title{
First Evidence for Family-Specific QTL for Temperature-Dependent Sex Reversal in Nile Tilapia (Oreochromis niloticus)
}

\author{
L.M. Lühmann a C. Knorr ${ }^{b} \quad$ G. Hörstgen-Schwark ${ }^{a}$ S. Wessels ${ }^{a}$ \\ ${ }^{a}$ Department of Animal Sciences, Division of Aquaculture and Water Ecology, and ${ }^{b}$ Division of Reproduction and \\ Biotechnology of Livestock, Göttingen, Germany
}

\section{Key Words}

$\mathrm{GSD} \cdot$ Nile tilapia $\cdot \mathrm{QTL} \cdot$ Sex determination $\cdot$ Sex reversal $\cdot$

Temperature $\cdot$ TSD

\begin{abstract}
This study for the first time screens microsatellite markers for associations with the temperature-dependent sex of Oreochromis niloticus. Previous studies revealed markers on linkage groups (LG) 1, 3, and 23 to be linked to the phenotypic sex of Oreochromis spp. at normal rearing temperatures. Moreover, candidate genes for sex determination and differentiation have been mapped to these linkage groups. Here, 6 families of a temperature-treated genetically all-female $(X X) F_{1}$-population were genotyped for 21 microsatellites on the 3 LGs. No population-wide QTL (quantitative trait loci) or marker trait associations could be detected. However, family-specific QTL were found on LG 1 flanked by UNH995 and UNH104, on LG 3 at the position of GM213, and on LG 23 next to GM283. Moreover, family-specific single marker associations for UNH995 and UNH104 on LG 1, GM213 on LG 3, as well as for UNH898 and GM283 on LG 23 were detected. Yet, marker trait associations could not explain the temperature-dependent sex of all fish in the respective families. The molecular cue for the temperature-dependent sex in Nile tilapia might partially coincide with allelic variants at major and mi-
\end{abstract}

nor genetic sex determining factors. Moreover, additional QTL contributing to variable liabilities towards temperature might persist on other LGs.

Copyright $\odot 2012$ S. Karger AG, Basel

The determination of sex in Nile tilapia (Oreochromis niloticus) is a very complex and not yet completely understood mechanism. Oreochromis niloticus has a genetic sex determination (GSD) system with male heterogamety (XX/XY) as the major genetic factor [Mair et al., 1991]. It is, however, postulated that further minor autosomal factors are able to override the major genetic sex determination [Mair et al., 1991; Müller-Belecke and HörstgenSchwark, 1995]. Furthermore, elevated temperatures of $34^{\circ} \mathrm{C}$ and more are also capable to switch the sex of genetically female (XX) Nile tilapia into phenotypic males [Baroiller et al., 1995a] if applied during the critical sensitive period when the gonads are still sexually undifferentiated [Baroiller et al., 1995a, b]. Tessema et al. [2006] postulated a highly effective temperature treatment for 10 days at $36^{\circ} \mathrm{C}$ between day 10 and 20 post fertilization (dpf). Temperature-dependent phenotypic sex is under genetic control. Male ratios in temperature-treated progenies are strongly dependent on the population and on the combination of the parental animals [Baroiller and

Liane-Magdalena Lühman

Department of Animal Sciences, Division of Aquaculture and Water Ecology Albrecht-Thaer-Weg 3 
D’Cotta, 2001; Tessema et al., 2006]. Moreover, it has been proven that temperature-dependent sex ratio is a heritable trait and it can be selected for as a quantitative trait. Wessels and Hörstgen-Schwark [2007, 2011] succeeded in the selection for high and low temperature response: within 3 generations of selection, the percentage of males in temperature-treated progeny groups of the 'high' and the 'low' line was $>92 \%$ and $<53 \%$, respectively. The cumulated realized heritability for the high and low line was 0.63 and 0.84 , respectively.

A comprehensive linkage map, derived from a $\mathrm{F}_{2}$-family of the hybrid cross $O$. niloticus $\times O$. aureus, exists for tilapia [Lee et al., 2005]. The map spans $1,311 \mathrm{cM}$ in 24 linkage groups (LG). It consists of 525 microsatellite markers and 21 gene-based markers. Associations with the phenotypic sex in Oreochromis spp. and interspecies crosses were reported for markers on LG 1, 3, and 23 [Shirak et al., 2002; Lee et al., 2003; Cnaani et al., 2004; Lee et al., 2004, 2005, 2011]. Furthermore, several candidate genes (amh, dmo, dmrta2, sox14, and $w t 1 b$ ) as putative master key regulators of sex determination in tilapia were in addition mapped to LG 23 [Lee et al., 2005; Shirak et al., 2006; Lee and Kocher, 2007; Cnaani et al., 2008].

In $O$. niloticus, the major sex determiner has been mapped to the chromosomal region between microsatellites GM201 and UNH104 on LG 1 (11 cM distance) [Lee et al., 2003; Lee and Kocher, 2007]. Later, Cnaani et al. [2008] mapped the major sex determiner to a $1 \mathrm{cM}$ region on the same chromosome flanked by UNH1O4 and UNH995. Recently, Eshel et al. [2011] described a strong association of the microsatellite UNH898 on LG 23 with the phenotypic sex in mixed sex (XX/XY) populations of O. niloticus. UNH898 has been mapped next to $a m h(1 \mathrm{cM}$ distance) [Shirak et al., 2006] which shows a strongly sex dimorphic expression in brains of $O$. niloticus at $14 \mathrm{dpf}$ shortly before the initial start of the gonadal differentiation [Poonlaphdecha et al., 2011]. Despite of the suggestive associations with the major sex determiner in different populations, the markers on the above mentioned LG 1,3 , and 23 do not explain the sex ratio in all investigated families. Thus, it is hypothesized that only the interaction of major and minor genetic factors together with temperature-dependent factors will contribute to finally explain the variety of sex ratios [Lee and Kocher, 2007; Cnaani et al., 2008].

There is no ample knowledge of genetic components contributing to temperature effects on the phenotypic sex in Nile tilapia. So far, a key role in temperature-dependent sex expression in Nile tilapia was only shown for cyp19a due to reduced expression in undifferentiated go-
Table 1. Number of males and females and male ratios in the control $\left(28^{\circ} \mathrm{C}\right)$ and temperature-treated $\left(36^{\circ} \mathrm{C}\right)$ groups of the genetically all-female $\mathrm{F}_{1}$-families

\begin{tabular}{llccc}
\hline Family & Treatment, ${ }^{\circ} \mathrm{C}$ & $\begin{array}{l}\text { Males } \\
(\Sigma=189)\end{array}$ & $\begin{array}{l}\text { Females } \\
(\Sigma=982)\end{array}$ & Male ratio, \% \\
\hline 1 & 28 & 0 & 107 & 0.0 \\
& 36 & 37 & 63 & 37.0 \\
\hline 2 & 28 & 0 & 101 & 0.0 \\
& 36 & 47 & 30 & 61.0 \\
\hline 3 & 28 & 0 & 108 & 0.0 \\
& 36 & 27 & 79 & 25.5 \\
\hline 4 & 28 & 0 & 95 & 0.0 \\
& 36 & 19 & 81 & 19.0 \\
\hline 5 & 28 & 0 & 96 & 0.0 \\
& 36 & 17 & 80 & 17.5 \\
\hline 6 & 28 & 2 & 94 & 2.1 \\
& 36 & 40 & 58 & 40.8 \\
\hline
\end{tabular}

nads of temperature-treated all-female fish [D'Cotta et al., 2001]. Cyp19a was mapped to LG 1, flanked by GM633 and UNH985 [Shirak et al., 2006; Lee and Kocher, 2007]. The evolutionary role, the development and the interdependence of GSD, temperature effects on the phenotypic sex, and temperature-dependent sex determination (TSD) are still under discussion [Sarre et al., 2004; Bull, 2008; Ospina-Alvarez and Piferrer, 2008; Shoemaker and Crews, 2009]. Bull [1980] proposed that GSD and TSD systems were mutually exclusive. In Nile tilapia, a coexistence of GSD and temperature effects on the phenotypic sex has been postulated [Wessels and HörstgenSchwark, 2007]. However, it is still questionable if a genetic correlation between GSD and temperature effects on the phenotypic sex persists. To test this hypothesis, 21 microsatellite markers on LG 1, 3, and 23 were investigated to prove any putative associations with the temperature-dependent sex of 180 temperature-treated fish derived from a cross between normal females (XX) and temperature sex-reversed males ( $\left.\Delta \_\mathrm{XX}\right)$.

\section{Materials and Methods}

Mapping Population

A genetically all-female (XX) mapping population of 6 genetically unrelated $\mathrm{F}_{1}$-families was established, derived from the Lake Manzala population (Egypt) (table 1). Six XX-females from the low line and 5 temperature sex-reversed $\Delta \delta \mathrm{XX}$ males from the high line originating from a previous selection experiment were 
Table 2. Characteristics of the chosen microsatellite markers with positions on the genetic map by Lee et al. [2005] and marker trait association statistics $\left(\chi^{2}, p\right)$ for the temperature-dependent phenotypic sex

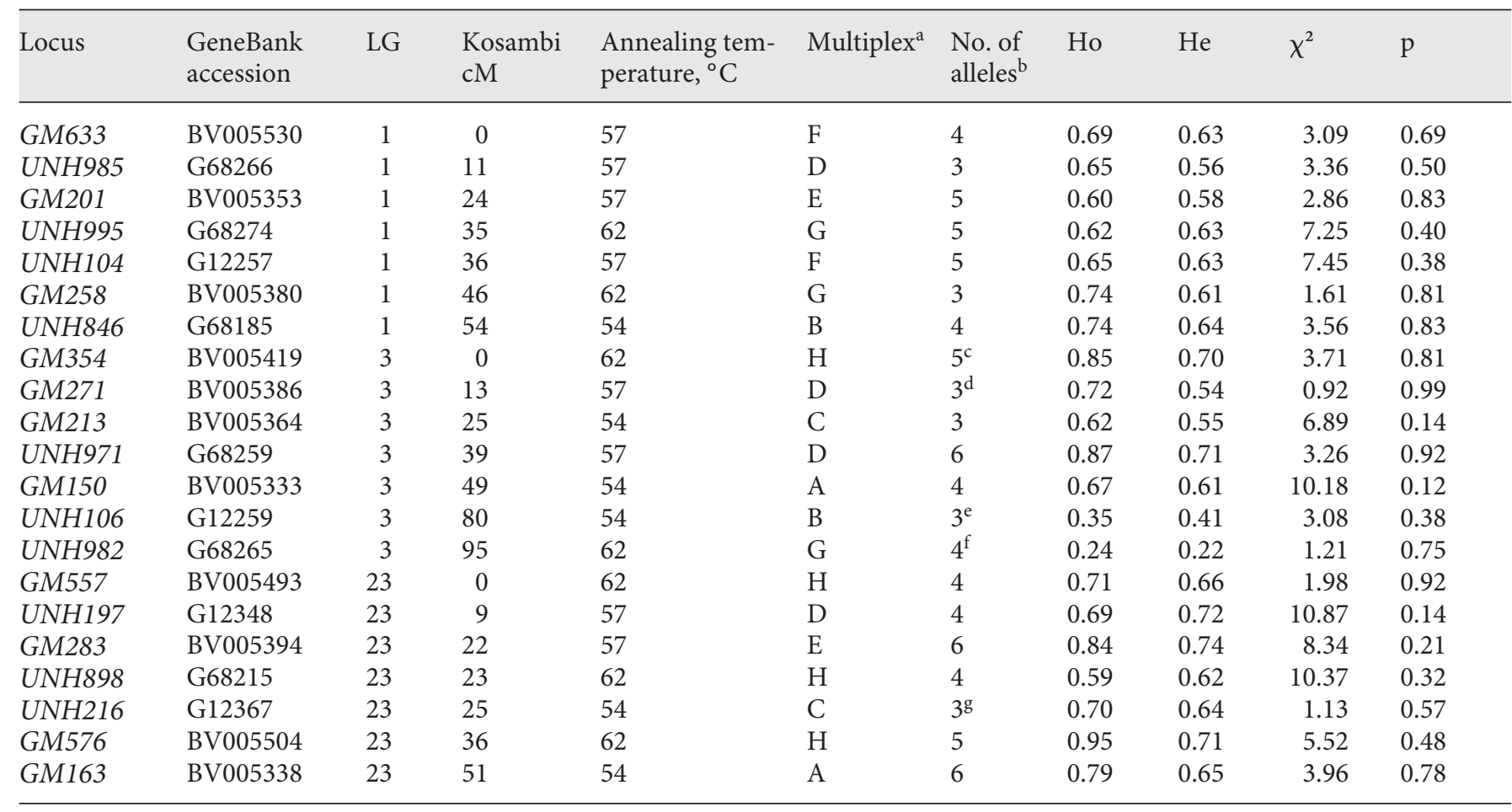

${ }^{a}$ Loci with the same capitals were used together in one PCR assay.

b Superscripts indicating null alleles.

${ }^{\mathrm{c}}$ Allele 96 in family 3; ${ }^{\mathrm{d}}$ allele 107 in family 6, allele 132 in families 1, 2, 3, 4; ${ }^{\mathrm{e}}$ allele 123 in family 2, allele 129 in family 3 ; ${ }^{\mathrm{f}}$ allele 102 in families 4 and 5, alleles 112 and 118 in family 2; ${ }^{g}$ allele 117 in families 4, 5, 6, allele 119 in families 4 and 5, allele 121 in families 1 and 3 .

used as parents [Wessels and Hörstgen-Schwark, 2007, 2011]. The temperature-dependent male ratios in the families of the low line females were $<60 \%$. In the sire families, the temperature-dependent male ratios were $>93 \%$. The males were previously tested for their genetic sex (XX/XY) by progeny testing [Lühmann et al., 2009], and fin clips were collected from each individual $(\mathrm{n}=11)$. For individual identification, all fish were tagged using passive integrated transponders. After artificial fertilization, the progeny groups were incubated for 10 days at $28^{\circ} \mathrm{C}$ until the larvae were split in 2 groups per family, each comprising 110 larvae. The control group was kept at $28^{\circ} \mathrm{C}$ and the temperature treatment was carried out at $36^{\circ} \mathrm{C}$ from days $10-20$ post fertilization as previously described by Tessema et al. [2006]. Temperature was checked 3 times a day. After 10 days of treatment, the groups were raised separately at $28^{\circ} \mathrm{C}$ for at least 2 months in mesh covered 80 -liter glass aquaria. Finally, 15 males and 15 females of each of the 6 temperature-treated groups were sexed alive by examination of the genital papilla, and fin clips were taken as DNA source. Surplus fish of both the temperature-treated groups and the control groups were killed by an overdose of anesthetics (diethylether), and the individual sex was microscopically examined using gonad squashes according to Guerrero and Shelton [1974].

QTL for Temperature-Dependent Sex Reversal in Nile Tilapia

\section{Genotyping of Microsatellite Markers}

The 21 microsatellite markers (table 2) used in this study were chosen from LGs 1, 3, and 23 published by Lee et al. [2005]. The selection of microsatellites aimed to achieve an even distribution over the linkage groups with an average distance of $9.5 \mathrm{cM}$ per LG.

DNA was isolated from the fin clips by phenol-chloroform extraction [Chomczynski and Sacchi, 1987]. Evaluation of microsatellites and allele calling were done on the ABI PRISM 3100 Genetic Analyzer with the ABI-software GENESCAN 3.7 and GENOTYPER 3.6 using GENESCAN ${ }^{\mathrm{TM}}-500 \mathrm{ROX}^{\mathrm{TM}}$ as internal size standards. Multiplex assays $(\mathrm{A}-\mathrm{H})$ of 2-4 primer pairs were optimized (table 2). PCR was carried out using $40 \mathrm{ng}$ of genomic DNA with $1 \times$ MultiplexMix (Qiagen, Hilden, Germany), $0.5 \times$ Q-Solution (Qiagen), 2.8-14 pmol of each primer (one labeled at the 5 -end alternatively with fluorescent dyes 6-FAM, CY3 or HEX) in a volume of $14 \mu \mathrm{l}$. The cycling conditions used were: 35 cycles at $92^{\circ} \mathrm{C}$ for $30 \mathrm{~s}$, a multiplex specific annealing temperature of $54^{\circ} \mathrm{C}, 57^{\circ} \mathrm{C}$, or $62^{\circ} \mathrm{C}$ for $30 \mathrm{~s}$, and an extension period of $35 \mathrm{~s}$ at $72^{\circ} \mathrm{C}$ with an initial denaturation for $5 \mathrm{~min}$ at $95^{\circ} \mathrm{C}$ and a final extension at $72^{\circ} \mathrm{C}$ for $5 \mathrm{~min}$. PCR reactions were performed using the Biometra T-Gradient thermocycler (Biometra, Göttingen, Germany). 
Statistical Analysis

For the examination of marker properties, the expected heterozygosity (also known as allelic diversity) $(\mathrm{He})$ and the observed heterozygosity $(\mathrm{Ho})$ were estimated for each microsatellite marker using the procedure ALLELE within the SAS/Genetics ${ }^{\circledR}$ software, version 9.2 (SAS Institute Inc, N.C., USA).

The linkage map for the genetically all-female (XX) $\mathrm{F}_{1}$-population was constructed using JOINMAP 4.0 (Kyazma, Wageningen, Netherlands) [Van Ooijen, 2006] under the cross population function. The Kosambi mapping function was applied with LOD scores of $\geq 3$. Maps of the 6 single $F_{1}$-families were constructed and merged to one integrated linkage map. The comparative map charts of the current linkage map and the reference mapping by Lee et al. [2005] was drawn using the software MapChart 2.1 [Voorrips, 2002]. Both linkage maps were compared by drawing homologous loci within MapChart 2.1.

The QTL (quantitative trait loci) mapping was carried out using GridQTL 2.1.5 [Seaton et al., 2006]. As a dependent variable, temperature-dependent phenotypic sex of the genetically all-female $\mathrm{F}_{1}$-population was considered as a binary trait, coded 1 for males and 2 for females. A chromosome-wide analysis, assuming a single QTL, was performed under the Visscher and Hopper [2001] option in $1 \mathrm{cM}$ intervals. The chromosome-wide significance thresholds for $\mathrm{p}=0.05$ and $\mathrm{p}=0.01$ were determined by bootstrapping with 1,000 iterations. Additionally, Pearson $\chi^{2}$ statistics (SAS/STAT ${ }^{\circledR}$ software, version 9.2 (SAS Institute Inc), Proc GLIMMIX) was performed to test for linkage between the single marker genotypes and the temperature-dependent phenotypic sex at the population and the family level. Families showing significant marker trait associations were analysed again for the respective marker genotypes by Fisher's exact test. Additionally, a family specific QTL mapping was performed with GridQTL 2.1.5 [Seaton et al., 2006] for the respective families using the same options as in the population wide QTL analysis described before.

\section{Results}

\section{Temperature-Dependent Phenotypic Sex of the}

Mapping Population

No phenotypic males were observed in the control groups, except for family 6 with 2 males out of 96 fish (2.1\%). Contrary to that, the temperature-treated groups showed 33.5\% males on average. The lowest percentage of males (17.5\%) was observed in family 5 , and the highest percentage of males $(61.0 \%)$ was observed in family 2 (table 1).

\section{Marker Properties}

In the parental generation, 2-6 alleles per locus were identified. Over all loci, the average number of alleles was 4.2 (table 2). In general, the chosen microsatellites were highly informative. A proportion of $2.1 \%$ missing alleles was found in the mapping population. The average $\mathrm{He}$ and Ho values were 0.61 and 0.68 , respectively. Two loci
(UNH106 and UNH982) showed Ho and He values $<0.5$ (table 2). Five of the 21 loci revealed up to 3 null alleles in specific families or in the whole mapping population (table 2). The remaining missing genotypes for these markers could be deduced from parental genotypes in most cases. For UNH982, the null alleles 112 and 118 were found in 1 family, with both alleles coming from the maternal genotype. It was not possible to deduce these alleles and they were set as missing values. For UNH216, the null alleles could not be deduced for family 6 and were thus set as missing values. Additionally, the genotyping of family 4 for UNH216 was insufficient. Only 5 males and 10 females were genotyped. Therefore, $1.2 \%$ of all missing alleles resulted from missing genotypes at the loci UNH216. However, $U N H 216$ has been included in all statistical analysis.

\section{Linkage Map}

GM633 and UNH985 remained unmapped, but an integrated linkage map consisting of 4 LGs (LOD $\geq 3$; fig. 1 ) was constructed. The total map length was $85.1 \mathrm{cM}$ with an average marker distance of $4.8 \mathrm{cM}$. LG 1 was $17.6 \mathrm{cM}$ long with 5 markers having an average distance of $4.4 \mathrm{cM}$. LG 3a consisted of 5 markers with an average distance of $7.9 \mathrm{cM}$ and a total length of $31.7 \mathrm{cM}$. LG $3 \mathrm{~b}$ included 2 markers with a distance of $1.1 \mathrm{cM}$. Seven markers were mapped to LG 23 over a total length of $34.7 \mathrm{cM}$ and an average marker distance of $5.8 \mathrm{cM}$.

\section{Marker Trait Associations for \\ Temperature-Dependent Phenotypic Sex}

The population wide QTL search for the phenotypic sex of the temperature-treated genetically all-female $\mathrm{F}_{1^{-}}$ population showed no significant QTL on any of the LGs (table 3). LOD scores were as high as 1.1 for LG 23 and as low as 0.03 for LG 3b. F-values were below the F-statistic thresholds for $\mathrm{p}=0.05$. Pearson $\chi^{2}$ test statistics for single marker associations did also not reveal significances at the population level (table 2). However, the family-based approach revealed several significant associations with the phenotypic sex: UNH995 and UNH104 on LG 1 in family 6, GM213 on LG 3a in family 2, and UNH898 (LG 23) and GM283 (LG 23) in family 4. Fisher's exact statistics for these 5 markers and the respective families are displayed in table 4. The subsequent QTL studies using GridQTL confirmed the associations for families 2, 4, and 6 (fig. 2-4).

Furthermore, segregation distortions for GM283 and UNH898 genotypes were found for family 4 . The 4 genotypes per marker significantly differed from the expected Mendelian segregation of 1:1:1:1 $(p<0.05)$. 


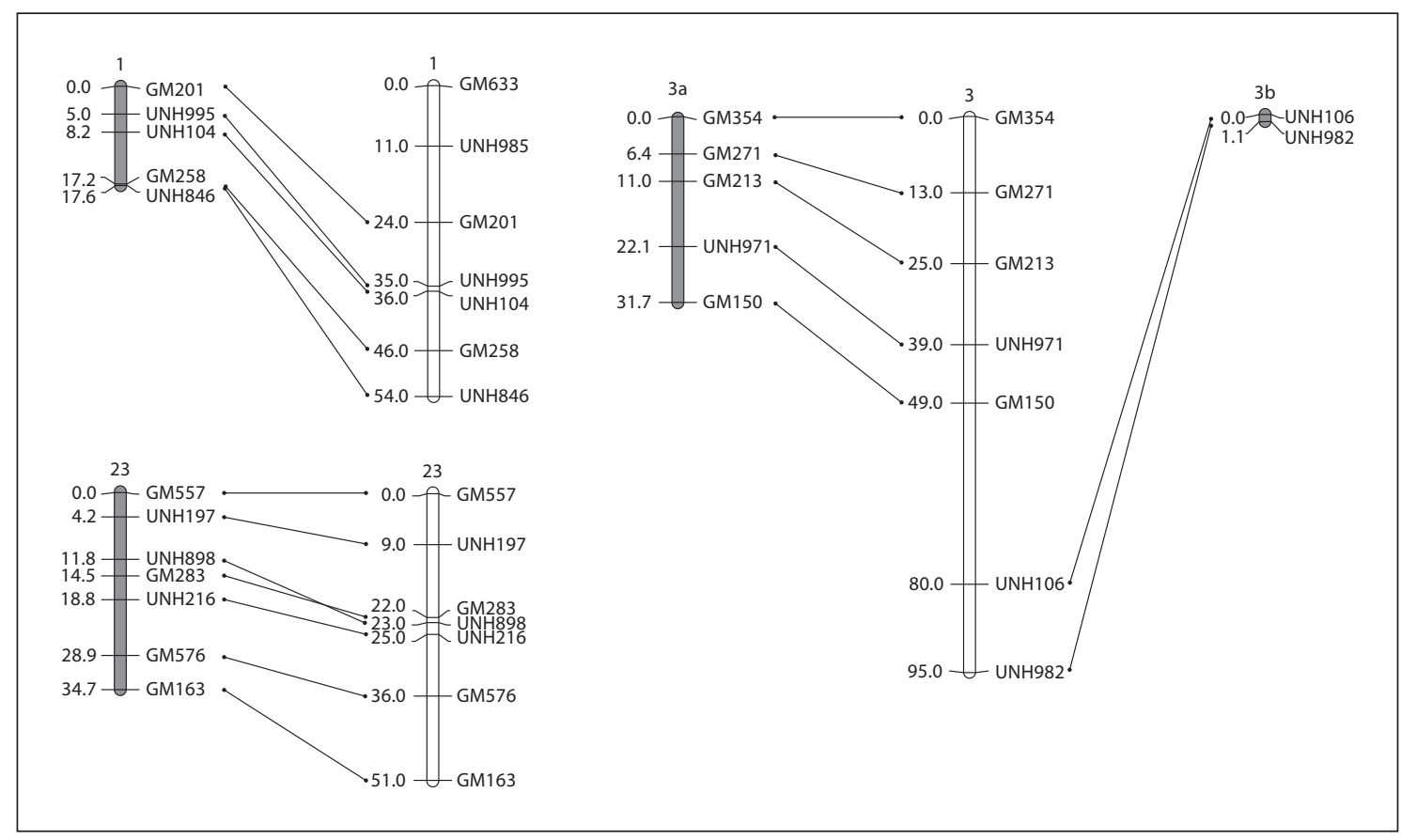

Fig. 1. Comparison of the genetic linkage map of Lee et al. [2005] (white) and the combined linkage map of 6 genetically all-female (XX) temperature-treated $\mathrm{F}_{1}$-families of $O$. niloticus (grey). Marker distances in Kosambi $\mathrm{cM}$ are given to the left of each linkage group.

\section{Discussion}

Previous studies have indicated that several genetic markers on LG 1, 3, and 23 are linked to the phenotypic sex of Oreochromis spp. [Shirak et al., 2002; Lee et al., 2003; Cnaani et al., 2004; Lee et al., 2004, 2005, 2011]. Moreover, different genes which are putatively involved in the sex differentiation have also been mapped to these linkage groups [Lee et al., 2005; Shirak et al., 2006; Lee and Kocher, 2007; Cnaani et al., 2008].

The current study is the first attempt to identify marker trait associations and QTL for the temperature-dependent phenotypic sex in a cichlid species with a sex determination system composed of major and minor genetic factors as well as clear temperature effects on the expression of the phenotypic sex.

\section{Phenotyping - Temperature-Dependent Phenotypic}

Sex

In order to assure that all males observed in the temperature-treated groups were temperature sex-reversed, a genetically all-female population was used in the present study. This genetically all-female population was derived from a cross of a high $(\Delta \triangleleft \mathrm{XX})$ and a low temperature sen-
Table 3. Population wide QTL statistics for temperature-dependent phenotypic sex in a genetically all-female (XX) population of O. niloticus

\begin{tabular}{clll}
\hline LG & F statistic & $\begin{array}{l}\text { F statistic threshold } \\
(\mathrm{p}=0.05)\end{array}$ & LOD \\
\hline 1 & 3.600 & 6.870 & 0.781 \\
$3 \mathrm{a}$ & 2.440 & 7.195 & 0.529 \\
$3 \mathrm{~b}$ & 0.130 & 5.291 & 0.029 \\
23 & 4.980 & 7.284 & 1.081 \\
\hline
\end{tabular}

sitive line (oXX) [Wessels and Hörstgen-Schwark, 2007, 2011]. In the current study, no males were observed in 5 of the 6 control groups of the mapping population. Thus, autosomal sex factors can be excluded in these families. However, in the control group of family 6, 2 males out of 96 fish were found which might result from a sex reversal caused by autosomal factors. A contamination of the control groups by other fish is very unlikely as all aquaria were mesh covered to prevent jumping over of fish. Nevertheless, all males in the temperature-treated groups of family 6 were assumed to be temperature sex-reversed. 


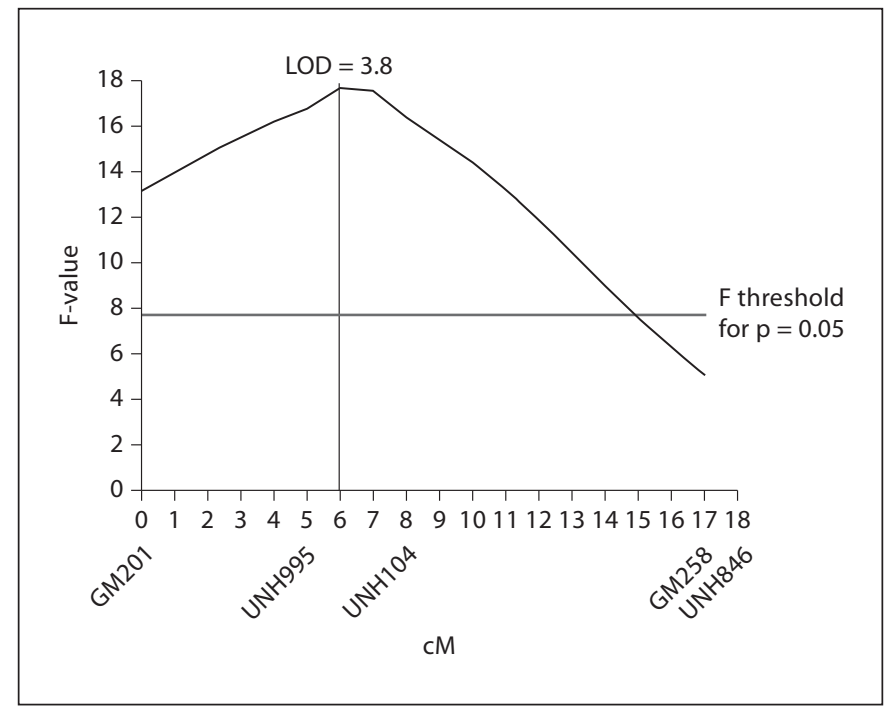

Fig. 2. QTL statistics for LG 1 within family 6 of the $F_{1}$-mapping population.

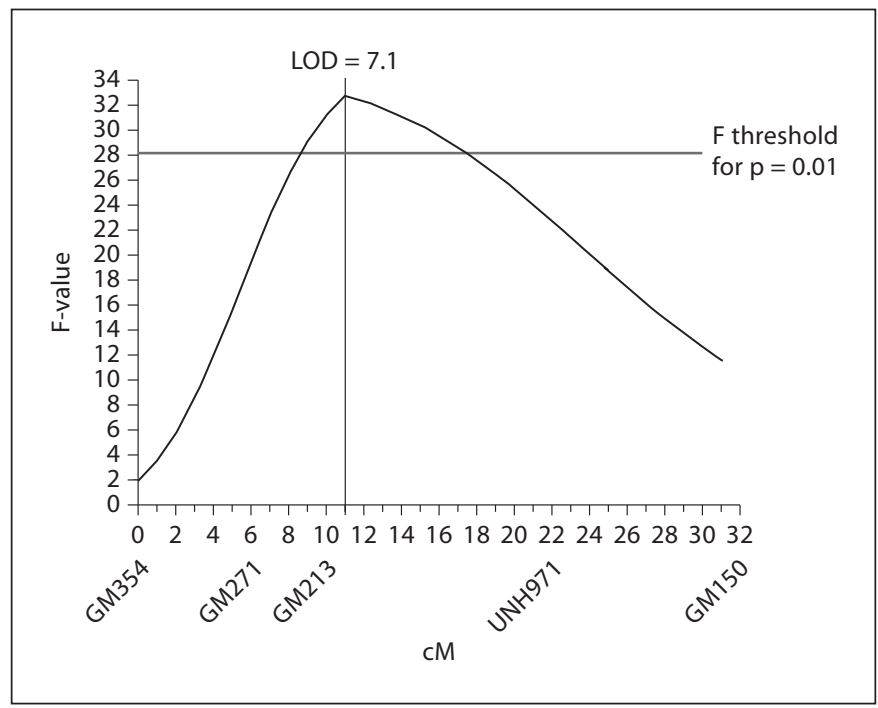

Fig. 3. QTL statistics for LG 3 a within family 2 of the $F_{1}$-mapping population.

Table 4. Number of males and females for each genotype of family-specific significantly associated loci with the temperature-dependent sex

\begin{tabular}{|c|c|c|c|c|c|c|c|}
\hline Locus & LG & Family $^{\mathrm{a}}$ & $\chi^{2}$ & $\mathrm{p}^{\mathrm{b}}$ & Genotype $^{c}$ & Males & Females \\
\hline UNH995 & 1 & $6(172 / 172 \times 172 / 220)$ & 6.65 & 0.025 & $172 / 220$ & 10 & 3 \\
\hline UNH104 & 1 & $6(129 / 129 \times 129 / 178)$ & 6.53 & 0.027 & $\begin{array}{l}129 / 178 \\
129 / 129\end{array}$ & $\begin{array}{r}11 \\
4\end{array}$ & $\begin{array}{r}4 \\
11\end{array}$ \\
\hline GM213 & $3 a$ & $2(76 / 83 \times 83 / 83)$ & 6.65 & 0.025 & $\begin{array}{l}76 / 83 \\
83 / 83\end{array}$ & $\begin{array}{r}10 \\
5\end{array}$ & $\begin{array}{r}3 \\
12\end{array}$ \\
\hline UNH898 & 23 & $4(254 / 274 \times 254 / 262)$ & 8.25 & 0.037 & $\begin{array}{l}254 / 274 \\
254 / 254 \\
262 / 274 \\
254 / 262\end{array}$ & $\begin{array}{r}1 \\
10 \\
1 \\
2\end{array}$ & $\begin{array}{l}4 \\
4 \\
6 \\
1\end{array}$ \\
\hline GM283 & 23 & $4(164 / 176 \times 176 / 178)$ & 8.68 & 0.026 & $\begin{array}{l}176 / 178 \\
164 / 176 \\
164 / 178 \\
176 / 176\end{array}$ & $\begin{array}{r}10 \\
2 \\
1 \\
2\end{array}$ & $\begin{array}{l}3 \\
7 \\
4 \\
1\end{array}$ \\
\hline
\end{tabular}

a The parental genotypes are given in brackets (dam $\times$ sire).

${ }^{\mathrm{b}}$ Fisher's exact probability.

${ }^{c}$ Allele sizes in basepairs.

\section{Marker Properties}

Within the $\mathrm{F}_{1}$-mapping population the microsatellites were highly informative. Both average values for Ho (0.68) and $\mathrm{He}(0.61)$ were moderately high, confirming their suitability for linkage and QTL mapping. Further, in the present study null alleles for several loci were ob- served (table 2). For GM271 also Cnaani and Kocher [2008] found null alleles in O. tanganicae, and they discussed that the occurrence of null alleles indicates developing sex chromosomes. This has also been described for stickleback species (Gasterosteidae) where null alleles were shown to occur sex specifically as a sign of sex chro- 


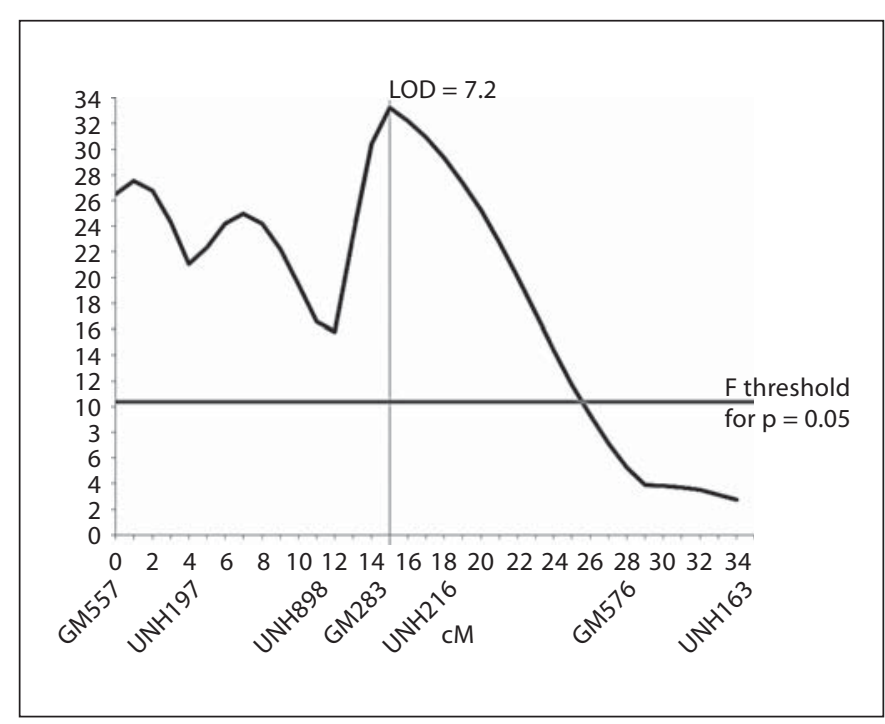

Fig. 4. QTL statistics for LG 23 within family 4 of the $F_{1}$-mapping population.

mosome differentiation [Ross et al., 2009; Shikano et al., 2011]. However, a sex-specific occurrence of null alleles could not be reported here.

\section{Linkage Mapping}

A comparison between the present linkage map and the reference map by Lee et al. [2005] revealed some differences. First of all, the linkage of GM633 and UNH985 to LG 1 could not be confirmed here (LOD $\leq 2$ ). Additionally, no linkage between both loci could be found in the present study; however, both markers were informative in the current mapping population. This lack of linkage may be due to the different fish species and populations used. Lee et al. [2005] worked with a species cross of $O$. niloticus and O. aureus whereas the present linkage map was constructed for an O. niloticus population. Additionally, the mapping population in the current study consisted of a genetically all-female (XX) population. The reported linkage of GM633 and UNH985 could thus have resulted from the mixed sex progeny of the crossed species.

Secondly, LG 3 was split in the present study, although the order of the loci persisted. Lee et al. [2005] reported a distance of $31 \mathrm{cM}$ between GM150 and UNH106. In the present investigation, the distance could even be larger as no linkage between the markers was observed leading to LG $3 \mathrm{a}$ and $\mathrm{LG} 3 \mathrm{~b}$.

Thirdly, on LG 23 the loci UNH898 and GM283 switched in order compared to the map calculated by Lee et al. [2005]. Again, species and population differences of the 2 studies might have attributed to the different results. Additionally, the current linkage groups (in cM) were shorter compared to the proposed length of the reference map (fig. 1). This may be due to the lower marker density for each linkage group as only a subset of the mapped markers was investigated in this study. Additionally, sex-specific recombination rates might explain the differences in LG lengths. Higher recombination rates in the telomeric region of males and in the centromeric region of females have been described for several fish species like rainbow trout and zebrafish [Sakamoto et al., 2000; Singer et al., 2002]. Also, Lee et al. [2004, 2005] observed different recombination rates for males and females in tilapia depending on the chromosomal region. Therefore, the current length of LGs could be specific for genetically female fish of O. niloticus.

\section{Marker Trait Associations for}

Temperature-Dependent Phenotypic Sex

It is still controversially discussed if loci responsible for temperature-dependent sex and GSD reside on the same linkage groups, if they are located closely to each other, or if they are basically identical.

Sarre et al. [2004] proposed a continuum of GSD and temperature-dependent phenotypic sex, with the same genes involved in both systems within a species. In this study, no QTL on the population level were identified, but some were mapped for families 2, 4, and 6. The QTL were in the majority localized in regions with known associations between the phenotypic sexes at normal temperature. In family 6 , significant associations to the temperature-dependent sex were found on LG 1 for the markers UNH995 $(5 \mathrm{cM})$ and UNH104 $(8.2 \mathrm{cM})$, confirmed by a significant QTL (LOD = 3.8) at the position of $6 \mathrm{cM}$ (fig. 2). For both markers the specific paternal alleles 220 (UNH995) and 178 (UNH104) were mostly found in males and might thus be suggested to be associated with phenotypic males after temperature treatment within this family. However, as not all of the male progenies possess these alleles, additional factors must control TSD in this family. In fact, the chromosomal region flanked by UNH995 and UNH104 harbors the major male sex determiner for O. niloticus [Cnaani et al., 2008]. One putative key regulator for sex determination, $w t 1 b$, has been mapped to LG 1, $5.9 \mathrm{cM}$ away from UNH995 and $6.6 \mathrm{cM}$ away from UNH104. But it has been excluded as the major male determiner [Lee and Kocher, 2007]. For tilapia, no temperature-dependent expression studies exist for $w t 1 b$. 
In family 2, GM213 on LG 3a was significantly associated with the phenotypic sex, confirmed by a significant QTL on LG 3a at position of $11 \mathrm{cM}$ (fig. 3). In this family, most female progenies possessed the identical-by-state microsatellite alleles of the sire, whereas the identical-bystate alleles of the dam were mostly found in male progenies. For O. aureus, a tilapia species with a female heterogametic sex determination system (ZZ/WZ), maternally inherited alleles of LG 3 markers are assumed to be sex-specific [Cnaani et al., 2008]. Additionally, dmo, which is involved in the gonadal development of the ovaries [Guan et al., 2000], has been mapped to the region between GM150 and UNH106 on LG 3, approximately 29 cM away from GM213 [Lee et al., 2005]. Because of the large interval, a higher marker density should be applied to detect putative associations with $d m o$.

Moreover, significant associations with UNH898 on LG 23 were found in family 4 (fig. 4). Three different alleles for UNH898 (254, 262, and 274) could be determined for this family. Progenies with the genotype 254/254 were male biased (10:4), whereas the genotype $254 / 274$ produced almost all-females (1:4). If allele 262 was present, no association with the phenotypic sex could be observed. Therefore, it might be assumed that each allele of UNH898 and their respective combinations represent a different liability to temperature within this family. Eshel et al. [2011] described the microsatellite marker UNH898 at LG 23 to be associated with the phenotypic sex of O. niloticus. The authors found allele 276 almost exclusively in males, and fish exhibiting genotypes with the alleles $253 / 253$, $253 / 274$, as well as $274 / 274$ were almost exclusively females. In the present study, allele 276 was not detected. This could be reasonable as a genetically all-female (XX) mapping population was used and no males were found in the control groups. However, allele sizes strongly depend on the method of microsatellite analysis, as respective sizes in bp can differ between the techniques. Here, alleles were not sequenced and might therefore not be completely comparable. Thus, individuals that are homozygous for allele 274 , respectively for allele 276 , should comparatively be sequenced to assess the degree of identity.

Strong associations were found for GM283 on LG 23 in family 4 . Ten out of 15 males showed the paternal genotype (176/178) whereas 12 out of 15 females were devoid of it. The QTL, detected for family 4, was mapped to $15 \mathrm{cM}(\mathrm{LOD}=7.2)$, close to $G M 283(14.5 \mathrm{cM})$. In family 4 , segregation distortion was found for the genotypes of both microsatellites GM283 and UNH898 on LG 23. Cnaani et al. [2008] also reported deviations from the expected Mendelian segregation for sex-linked markers on LG 1 in O. aureus and O. mossambicus. The authors concluded that lethal alleles were linked to the sex determining factor. In the current mapping population no differences in mortality in dependence to the combination of alleles were detected after the 9th dpf. If lethal alleles caused the segregation distortion in family 4 , they would have had an impact on the survival before the 9 th dpf.

Different putative master key regulators for sex determination have been mapped to LG 23 [Shirak et al., 2002, 2006; Cnaani et al., 2007]. Amh has been mapped to the position of GM283, just $1 \mathrm{cM}$ away from UNH898 [Shirak et al., 2002]. Although amh exhibited a sex-specific expression in brains of $14 \mathrm{dpf}$ old O. niloticus fry, there is no definite proof that the expression of $a m h$ is also temperature-dependent [D'Cotta et al., 2007]. Within other species, the role of amh in TSD is controversially discussed. Amh is involved in the regulation of cyp19a expression [Josso et al., 1998]. Therefore, a temperature-related expression of $a m h$ could be the result of a feedback effect of cyp19a expression [Pieau, 1996].

Shirak et al. [2006] and Lee and Kocher [2007] mapped cyp19a to LG 1 between the microsatellites GM633 and UNH985. Both microsatellites could not be mapped to the present linkage map (fig. 1). Therefore, the QTL study could not cover this chromosomal region, and no QTL or single marker-trait association could be found here. To achieve linkage in further experiments, a higher density of markers especially in this region should be realized.

No significant QTL or single marker association could be found for any of the markers in families 1, 3, and 5 . Additionally, significant marker trait associations could not explain the temperature-dependent sex of all fish in the respective families. Therefore, it can be assumed that additional family-specific QTL for the temperature-dependent phenotypic sex might be found on other LGs as well. D'Cotta et al. [2007], for example, provided a first evidence for a temperature-dependent expression of sox 9 in $O$. niloticus, resulting in an earlier increase of sox 9 expression in XX-gonads compared to XY-gonads. Moreover, dmrt1 expression is known to be up-regulated by male producing temperature in different TSD-species like Trachemys scripta, Lepidochelys olivacea, and Oryzias latipes [e.g. Kettlewell et al., 2000; Torres et al., 2002; Hattori et al., 2007]. Dmrt1 has been mapped to LG 12 in tilapia [Lee et al., 2005]. Hence, a genome-wide approach with special emphasis on chromosomal regions harboring candidate genes for sex determining processes seems to be promising. 
The QTL detected herein correspond to regions known for sex determination at normal rearing temperatures. Summarizing the controversial discussion about the interdependence of genetic and temperature effects on sex determination of vertebrates, Grossen et al. [2011] suggested for species with GSD that the temperature effect is hidden by genes with major effects. But a certain threshold of liability values has to be exceeded [Grossen et al., 2011]. This threshold model could also be suggested for the sex determination process in $O$. niloticus. A certain number of temperature-responsive allelic variants have to be exceeded to result in phenotypic male differentiation during temperature treatments. This might be the reason why not all progenies become male in a temperature treatment. Multiple interacting loci have also been postulated for autosomal sex determination in Nile tilapia [Müller-Belecke and Hörstgen-Schwark, 1995; Ser et al., 2010], yet the number of underlying QTL in both systems of sex determination remains to be determined. Therefore, temperature-dependent sex expression in Nile tilapia might rather be a result of a polygenic system causing several liability values which might overlap with liability values for GSD.

This is further underlined when looking at the allele inheritance of the significant markers in family 6 in which the sex determining allele came from the sire and was located on LG 1 . Therefore, factors influencing temperature and genetic effects on sex determination might at least partially reside in the same major sex determining region (LG 1), also showing patterns of inheritance comparable to the XX/XY system for GSD. In contrast to that the sex determining allele of GM213 in family 2 came from the dam and was located on LG 3 like in a WZ/ZZ system. The inheritance of sex determining alleles from LG 3 has never been described before for O. niloticus. However, studies about sex linked markers on LG 1 in $O$. niloticus reported missing linkage for markers on LG 1
[Lee et al., 2003; Ezaz et al., 2004]. The sex determining loci on LG 3 may be an evolutionary rudiment, because LG 3 has been suggested to be the ancestral sex chromosome and that sex determination loci have shifted to LG 1 [Cnaani et al., 2008]. Shifts of sex determining loci from the ancestral sex chromosome to other LGs could also be the reason for the detected linkage of UNH898 and GM283 on LG 23 in family 4. Temperature effects on sex determination (and their absence) might play a crucial role in evolution and fixation of novel sex chromosomes or major sex factors as observed in Nile tilapia on LG 1.

Moreover, only a single QTL has been found per family. Additionally, the QTL detected here were different for the families. Therefore, the chromosomal regions or genes on which the temperature acts seem to be dependent on the family. Because of the high variability of significant markers and QTL for the different families, it can be assumed that the present mapping population will provide an excellent basis for further studies. Furthermore, the analysis of candidate genes is very promising, especially of amh and cyp19a. For both genes, polymorphisms have already been described for Oreochromis spp. [Shirak et al., 2006]. Additionally, the microsatellite markers mostly show a high informativeness in this population which increases their suitability for further experiments. A following $\mathrm{F}_{2}$-population will provide additional segregations of the markers and QTL, including further LGs. Moreover, a higher accuracy and confirmation of the QTL positions is aimed to be achieved by using more markers in the current QTL regions.

\section{Acknowledgements}

The authors would like to thank Sabrina Pach and Birgit Reinelt for their technical assistance. This work was supported by the German Research Foundation (WE 4434/2-1).

\section{References}

Baroiller JF, D’Cotta H: Environment and sex determination in farmed fish. Comp Biochem Physiol C Toxicol Pharmacol 130:399409 (2001).

- Baroiller JF, Chourrout D, Fostier A, Jalabert B: Temperature and sex-chromosomes govern sex ratios of the mouthbrooding cichlid fish Oreochromis niloticus. J Exp Zool 273:216223 (1995a).

QTL for Temperature-Dependent Sex

Reversal in Nile Tilapia
Baroiller JF, Clota F, Geraz E: Temperature sex Bull JJ: Sex determination: are two mechanisms determination in two tilapia Oreochromis niloticus and the red tilapia (Red florida strain): effect of high or low temperature, in Goetz FW, Thomas P (eds): Proceedings of the Fifth International Symposium on the Reproductive Physiology of Fish, pp 158-160 (University of Texas, Austin 1995b).

Bull JJ: Sex determination in reptiles. Q Rev Biol 55:3-21 (1980). better than one? J Bioscience 33:5-8 (2008).

Cnaani A, Kocher TD: Sex-linked markers and microsatellite locus duplication in the cichlid species Oreochromis tanganicae. Biol Lett 4:700-703 (2008).

Cnaani A, Zilberman N, Tinman S, Hulata G, Ron M: Genome-scan analysis for quantitative trait loci in an $\mathrm{F}_{2}$ tilapia hybrid. Mol Genet Genom 272:162-172 (2004). 
-Cnaani A, Lee BY, Ozouf-Costaz C, Bonillo C, Baroiller JF, et al: Mapping of sox2 and sox 14 in tilapia (Oreochromis spp.). Sex Dev 1:207210 (2007).

-Cnaani A, Lee BY, Zilberman N, Ozouf-Costaz C, Hulata G, et al: Genetics of sex determination in tilapiine species. Sex Dev 2:43-54 (2008).

Chomczynski P, Sacchi N: Single-step method of RNA isolation by acid guanidinium thiocyanate-phenol-chloroform extraction. Anal Biochem 162:156-159 (1987).

D'Cotta H, Fostier A, Guiguen Y, Govoroun M, Baroiller JF: Aromatase plays a key role during normal and temperature-dependent sex differentiation of tilapia Oreochromis niloticus. Mol Reprod Dev 59:265-276 (2001).

D'Cotta H, Pepey E, Pfennig F, Bienvenu D, Gutzeit HO, et al: Sox 9a, Sox9b and Amh are up-regulated in the gonads during natural and temperature-induced tilapia male differentiation, in Roudaut G, Labbé C, Bobe J (eds): Proceedings of the 8th International Symposium on Reproductive Physiology of Fish, pp 40 (Saint-Malo, France 2007).

-Eshel O, Shirak A, Weller JI, Slossman T, Hulata $\mathrm{G}$, et al: Fine-mapping of a locus on linkage group 23 for sex determination in Nile tilapia (Oreochromis niloticus). Anim Genet 42: 222-224 (2011).

-Ezaz MT, Harvey SC, Boonphakdee C, Teale AJ, McAndrew BJ, Penman DJ: Isolation and physical mapping of sex-linked AFLP markers in Nile tilapia (Oreochromis niloticus L.). Mar Biotechnol 6:435-445 (2004).

Grossen C, Neuenschwander S, Perrin N: Temperature-dependent turnovers in sex-determination mechanisms: a quantitative model. Evolution 65:64-78 (2011).

-Guan G, Kobayashi T, Nagahama Y: Sexually dimorphic expression of two types of DM (Doublesex/Mab-3)-Domain genes in a teleost fish the tilapia (Oreochromis niloticus). Biochem Bioph Res Co 272:662-666 (2000).

-Guerrero RD, Shelton WL: Aceto-carmine squash method for sexing juvenile fishes. Prog Fish Cult 36:56-56 (1974).

- Hattori RS, Gould RJ, Fujioka T, Saito T, Kurita J, et al: Temperature-dependent sex determination in Hd-rR Medaka Oryzias latipes: gender sensitivity, thermal threshold critical period and DMRT1 expression profile. Sex Dev 1:138-146 (2007).

-Josso N, Racine C, di Clemente N, Rey R, Xavier $\mathrm{R}$ : The role of anti-Müllerian hormone in gonadal development. Mol Cell Endocrinol 145:3-7 (1998).

-Kettlewell JR, Raymond CS, Zarkower D: Temperature-dependent expression of turtle Dmrt1 prior to sexual differentiation. Genesis 26:174-178 (2000).

Lee BY, Kocher TD: Exclusion of Wilms tumor (WT1b) and ovarian cytochrome P450 aromatase (CYP19A1) as candidates for sex determination genes in Nile tilapia (Oreochromis niloticus). Anim Genet 38:85-86 (2007).

Seaton G, Hernandez J, Grunchec JA, White I, Allen J, et al: GridQTL: a Grid Portal for QTL mapping of compute intensive datasets. Proceedings of the 8th World Congress on Genetics Applied to Livestock Production. (Belo Horizonte, Brazil 2006). of a sex-determining region in Nile tilapia (Oreochromis niloticus) using bulked segregant analysis. Anim Genet 34:379-383 (2003).

Lee BY, Hulata G, Kocher TD: Two unlinked loci controlling the sex of blue tilapia (Oreochromis aureus). Heredity 92:543-549 (2004).

-Ser JR, Roberts RB, Kocher TD: Multiple interacting loci control sex determination in Lake Malawi cichlid fish. Evolution 64:486-501 (2010).

Howe AE et al: A second-generation genetic linkage map of tilapia (Oreochromis spp.). Genetics 170:237-244 (2005).

Lee BY, Coutanceau JP, Ozouf-Costaz C, D'Cotta $\mathrm{H}$, Baroiller JF, Kocher TD: Genetic and physical mapping of sex-linked AFLP markers in Nile tilapia (Oreochromis niloticus). Mar Biotechnol 13:557-562 (2011).

Lühmann L, Samavati S, Wessels S, HörstgenSchwark G: The effect of temperature and hormone treatments during embryonic and post-larvae stages on sex differentiation in Nile tilapia (Oreochromis niloticus). Book of Abstracts of 10th International Symposium on Genetics in Aquaculture Bangkok, Thailand. 2009:217 (2009).

- Mair GC, Scott AG, Penman DJ, Beardmore JA, Skibinski DOF: Sex determination in the genus Oreochromis. 1. Sex reversal gynogenesis and triploidy in O. niloticus (L.). Theor Appl Genet 82:144-152 (1991).

Müller-Belecke A, Hörstgen-Schwark G: Sex determination in tilapia (Oreochromis niloticus) sex ratios in homozygous gynogenetic progeny and their offspring. Aquaculture 137:57-65 (1995).

Ospina-Alvarez N, Piferrer F: Temperature-dependent sex determination in fish revisited: prevalence, a single sex ratio response pattern, and possible effects of climate change. PLoS One 3:e2837 (2008).

Pieau C: Temperature variation and sex determination in reptiles. BioEssays 18:19-26 (1996).

Poonlaphdecha S, Pepey E, Huang SH, Canonne $\mathrm{M}$, Soler L, et al: Elevated $a m h$ gene expression in the brain of male tilapia (Oreochromis niloticus) during testis differentiation. Sex Dev 5:33-47 (2011).

Ross JA, Urton JR, Boland J, Shapiro MD, Peichel CL: Turnover of sex chromosomes in the stickleback fishes (Gasterosteidae). PLoS Genet 5:e1000391 (2009).

-Sakamoto T, Danzmann RG, Gharbi K, Howard P, Ozaki A, et al: A microsatellite linkage map of rainbow trout (Oncorhynchus mykiss) characterized by large sex-specific differences in recombination rates. Genetics 155 : 1331-1345 (2000).

- Sarre SD, Georges A, Quinn A: The ends of a continuum: genetic and temperature-dependent sex determination in reptiles. Bioessays 26:639-645 (2004).
Shikano T, Natri HM, Shimada Y, Merilä J: High degree of sex chromosome differentiation in stickleback fishes. BMC Genomics 12:474 (2011).

Shirak A, Palti Y, Cnaani A, Korol A, Hulata G, et al: Association between loci with deleterious alleles and distorted sex ratios in an inbred line of tilapia (Oreochromis aureus). J Hered 93:270-276 (2002).

-Shirak A, Seroussi E, Cnaani A, Howe AE, Domokhovsky R, et al: Amh and dmrta2 genes map to tilapia (Oreochromis spp.) linkage group 23 within quantitative trait locus regions for sex determination. Genetics 174: 1573-1581 (2006).

-Shoemaker CM, Crews D: Analyzing the coordinated gene network underlying temperature-dependent sex determination in reptiles. Semin Cell Dev Biol 20:293-303 (2009).

- Singer A, Perlman H, Yan Y, Walker C, CorleySmith G, et al: Sex-specific recombination rates in zebrafish (Danio rerio). Genetics 160: 649-657 (2002).

Tessema M, Müller-Belecke A, HörstgenSchwark G: Effect of rearing temperatures on the sex ratios of Oreochromis niloticus populations. Aquaculture 258:270-277 (2006).

Torres Maldonado LC, Landa Piedra A, MorenoMendoza N, Marmolejo Valencia A, Meza Martinez A, Merchant-Larios H: Expression profiles of Dax1, Dmrt1, and Sox9 during temperature sex determination in gonads of the sea turtle Lepidochelys olivacea. Gen Comp Endocrinol 129:20-26 (2002).

Van Ooijen JW: JoinMap ${ }^{\circledR} 4$ Software for the calculation of genetic linkage maps in experimental populations. Kyazma B.V. Wageningen Netherlands (2006).

-Visscher PM, Hopper JL: Power of regression and maximum likelihood methods to map QTL from sib-pair and DZ twin data. Ann Hum Genet 65:583-601 (2001).

Voorrips RE: MapChart: software for the graphical presentation of linkage maps and QTLs. J Hered 93:77-78 (2002).

-Wessels S, Hörstgen-Schwark G: Selection experiments to increase the proportion of males in Nile tilapia (Oreochromis niloticus) by means of temperature treatment. Aquaculture 272:S80-S87 (2007).

Wessels S, Hörstgen-Schwark G: Temperaturedependent sex ratios in selected lines and crosses with a YY-male in Nile tilapia (Oreochromis niloticus). Aquaculture 318:79-84 (2011). 